\title{
Extraosseous Ewing sarcoma presenting as recurrent intergluteal pilonidal disease
}

\author{
Marisa A. Sewell ${ }^{1,2^{*}}$ (D) Frieda M. Hulka ${ }^{2}$ and Jacob P. Zucker ${ }^{3}$
}

\begin{abstract}
Background: Pilonidal disease classically presents as an abscess or soft tissue swelling which classically occurs in the intergluteal cleft, just above the anus. Risk factors for this disease include obesity, prolonged sitting, and abundance of gluteal hair. Treatment options are extensive but most often include incision and drainage with antibiotic treatment. Presentation of recurrent intergluteal pilonidal disease in a young, active female would then be unusual. We present a case of a 17-year-old female presenting with what appeared to be intergluteal pilonidal disease. Definitive excision with histopathology revealed a diagnosis of extraosseous Ewing sarcoma.

Case presentation: An otherwise healthy and active 17-year-old female presented to our Pediatric Oncology clinic with a 2-month history of recurrent painful soft tissue swelling of the intergluteal cleft. At that time, she had been diagnosed with pilonidal abscess and had already undergone three incision and drainage procedures. A definitive excision with pathology was performed within weeks of her initial presentation. Immunohistochemical evaluation confirmed a diagnosis of extraosseous Ewing sarcoma.

Conclusion: This unusual case underlies the importance of considering a broad differential when evaluating potential pilonidal abscess in a patient who otherwise has no risk factors. Additionally, definitive excision with pathology is critical in a patient with unusually recurrent disease as this can be crucial in the identification of an alternative, and potentially devastating, diagnosis.
\end{abstract}

Keywords: Ewing sarcoma, Pilonidal disease, Abscess

\section{Background}

Pilonidal disease presents in people of all ages as an itchy, painful soft tissue swelling in the intergluteal cleft often just above the anus [1]. The mechanism of development is not clear but risk factors include obesity, prolonged sitting, and presence of large amounts of hair in the gluteal cleft. This disease is most often treated with incision and drainage but can also be definitively excised with reconstruction [2]. The differential of this disease is extensive; however, to our knowledge, this is the first published case of extraosseous Ewing sarcoma presenting as an intergluteal cleft mass. We present a case of an otherwise healthy

\footnotetext{
* Correspondence: marisasewell@gmail.com

'School of Medicine, University of Nevada Reno, 1664 N Virginia Ave, Reno, NV 89557-0357, USA

${ }^{2}$ Department of Surgery, School of Medicine, University of Nevada Reno, 1664 N Virginia Ave, Reno, NV 89557-0357, USA

Full list of author information is available at the end of the article
}

17-year-old female with a 6-month history of recurrent intergluteal "pilonidal abscess" which was excised and found to be extraosseous Ewing sarcoma.

\section{Case presentation}

This patient is an otherwise active and healthy female who presented at age 17 with a 2-month history of a worsening pruritic and painful swelling in the superior gluteal cleft. She presented to an outpatient clinic where she was diagnosed with pilonidal abscess and underwent local incision and drainage. The aspirate at that time was culture negative with no antibiotics given. The swelling recurred quickly, and she underwent a second local incision and drainage one week later. It recurred a third time, at which time she underwent total excision. Local pathology reports at that time indicated a nonspecific neoplasm; samples were sent to a consulting 
pediatric pathologist and were found to be CD99 and PAX7 positive. FISH analysis finalized the diagnosis of extraosseous Ewing sarcoma. PET imaging at that time was significant for FDG activity in the sacrococcygeal soft tissue with extension into unilateral gluteus minimus muscle. There was no evidence of bony tumor or metastasis.

Soon after definitive diagnosis, the patient presented for induction chemotherapy. At that time, the mass had already grown significantly, perhaps due to unroofing during her initial excision with biopsy (Fig. 1). The patient responded well to 10 weeks of induction chemotherapy (vincristine, doxorubicin, cyclophosphamide, etoposide, ifosfamide) with considerable reduction in tumor size. After induction chemotherapy, the patient underwent definitive sacrococcygeal and gluteal excision with wide margins with immediate reconstruction via rotational advancement gluteal flap. Post-operative pathology showed negative margins.

After her procedure, the patient suffered from prolonged wound healing and post-surgical abscess formation along the incision site positive for multi-drug resistant Klebsiella pneumoniae. Otherwise, the patient recovered well and then completed 22 weeks of consolidation chemotherapy. This was complicated by an episode of sepsis complicated by Klebsiella bacteremia and neutropenia. She had several episodes of neutropenic fever and pancytopenia but otherwise tolerated her treatment well. Since completion of her therapy to the time of publication of this manuscript, her physical exam and imaging have shown no evidence of disease recurrence.

\section{Conclusion}

Pilonidal disease describes an inflammatory phenomenon often in the intergluteal cleft causing the formation of small open "pits," which can then become secondarily infected and can cause formation of abscesses and fulminant cellulitis [1]. Risk factors for this disease include obesity, high density of hair in the intergluteal region, local trauma, and sedentary lifestyle [2]. Therefore, presentation of this disease in a healthy, active young person with no risk factors (such as our patient) would be unusual [1].

There are several approaches to treatment of this disease. Surgical treatment can include incision and drainage, marsupialization, curettage, and up to en bloc resection with reconstruction for definitive treatment [2]. Surgical treatment is often complicated by recurrence, surgical site infection, and poor wound healing [2]. It would not be unusual, then, for a surgeon to have to treat a pilonidal sinus abscess more than once.

In this case report, we describe a young female who presented with what appeared to be non-classic pilonidal sinus disease. This required multiple incision and drainage procedures culminating in total excision with gluteal flap all before a diagnosis of extraosseus Ewing sarcoma was established. The need for repeated treatment of her disease was not alarming at the time due to the relapsing nature of classic pilonidal disease. In retrospect, however, this patient had no risk factors for pilonidal disease. She was a healthy, active, fit young person presenting with a disease that normally affects those who are sedentary and have an abundance of hair in the gluteal cleft.

The differential for pilonidal sinus disease is extensive. It includes other infectious processes such as cryptoglandular abscess and hidradenitis, anorectal fistula, and inflammatory bowel disease [1]. More unusual diagnoses that can present as pilonidal disease include manifestations of systemic disease such as syphilis and actinomycosis. More rare still are unusual presentations of some

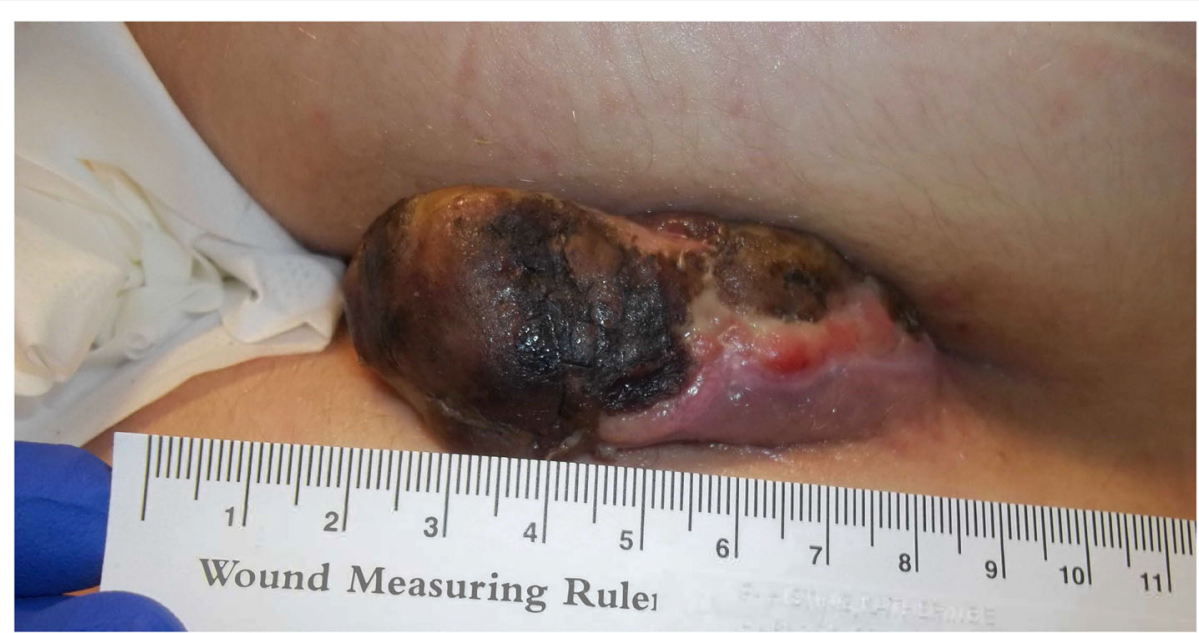

Fig. 1 Photo of intergluteal sacral tumor, taken at the time of start of induction chemotherapy 
cancers including sacrococcygeal teratoma, and in our case, soft tissue Ewing sarcoma [3].

Extraosseous Ewing sarcoma presenting in the intergluteal cleft has not previously been described. Ewing sarcoma is a neoplasm of soft tissue or bone, which primarily affects children and young adults [4]. It most commonly presents in males and as a painful swelling in the pelvis or in the epiphysis of long bones. Ewing sarcoma can uncommonly present in an extraosseous location as a soft tissue mass; when it does, it has no specific clinical manifestation beyond pain and swelling in the tumor site [5]. This tumor is often mistaken as an injury from playing a sport, for growing pains, or is found on imaging for workup of another diagnosis. Gold standard diagnosis is with biopsy followed by histopathology for tumor markers.

This case is significant as it is the first described case of extraosseous Ewing sarcoma presenting as an intergluteal cleft mass. Though this presentation is quite rare, it is important for surgeons to consider a broad differential when presented with recurrent pilonidal disease, including an unusual presentation of a neoplasm. This is especially critical in cases like ours in which patients with no classic risk factors for pilonidal disease present with an unusual gluteal swelling. This case underlies the importance of definitive excision with histological evaluation in unusual cases; our patient has made a full recovery from her Ewing sarcoma likely because it was identified and treated early in the disease course.

\section{Acknowledgements}

Not applicable.

\section{Authors' contributions}

As the corresponding author, I can attest that this manuscript has been seen and approved by all authors. All authors, including $\mathrm{FH}, \mathrm{JZ}$, and MS, contributed to this case and writing the subsequent manuscript. All authors read and approved the final draft of this work.

\section{Funding}

The authors did not receive support from any organization for the submitted work.

Availability of data and materials

Available upon request.

Ethics approval and consent to participate

Not applicable to this study.

\section{Consent for publication}

Our patient gave written consent to participate in this case report with the understanding that the intent was to publish these findings.

\section{Competing interests}

The authors declare they have no competing interests.

\section{Author details}

${ }^{1}$ School of Medicine, University of Nevada Reno, 1664 N Virginia Ave, Reno, NV 89557-0357, USA. ²Department of Surgery, School of Medicine, University of Nevada Reno, 1664 N Virginia Ave, Reno, NV 89557-0357, USA.

${ }^{3}$ Department of Pediatric Hematology/Oncology, Renown Health Children's Hospital, 1155 Mill St, Reno, NV 89502, USA.
Received: 28 September 2020 Accepted: 29 November 2020

Published online: 21 December 2020

References

1. Khanna A, Rombeau JL. Pilonidal disease. Clin Colon Rectal Surg. 2011;24(1): 46-53. https://doi.org/10.1055/s-0031-1272823.

2. "The management of pilonidal disease: a systematic review- ClinicalKey." https://www-clinicalkey-com.unr.idm.oclc.org/\#!/content/playContent/1-s2.0S0022346819302027? returnurl=null\&referrer=null (Accessed 15 Jul 2020).

3. Kumar N, Khosla D, Kumar R, Saikia UN, Singh S. Sacro-coccygeal teratoma in adult: two rare case reports and review of literature. Int J Appl Basic Med Res. 2014;4(2):122-4. https://doi.org/10.4103/2229-516X.136803.

4. Grünewald TGP, Cidre-Aranaz F, Surdez D, et al. Ewing sarcoma. Nat Rev Dis Primers. 2018;4:5. https://doiorg.unr.idm.oclc.org/10.1038/s41572-018-0003-X.

5. Galyfos G, Karantzikos GA, Kavouras N, Sianou A, Palogos K, Filis K Extraosseous Ewing sarcoma: diagnosis, prognosis and optimal management. Indian J Surg. 2016;78(1):49-53. https://doi.org/10.1007/ s12262-015-1399-0.

\section{Publisher's Note}

Springer Nature remains neutral with regard to jurisdictional claims in published maps and institutional affiliations.

\section{Submit your manuscript to a SpringerOpen ${ }^{\odot}$ journal and benefit from:}

- Convenient online submission

- Rigorous peer review

- Open access: articles freely available online

- High visibility within the field

- Retaining the copyright to your article

Submit your next manuscript at $\boldsymbol{\nabla}$ springeropen.com 